\title{
The Effects of Production Parameters on the Core Visibility Ratio of Dual-Core Yarns
}

\author{
Gamze Kılıç ${ }^{1}$, Bekir Yıldırım¹, Halil İbrahim Çelik², Sümeyye Üstüntağ ${ }^{1}$, Hüseyin Gazi Türksoy ${ }^{1}$ \\ ${ }^{1}$ Department of Textile Engineering, Erciyes University, Kayseri, Turkey \\ ${ }^{2}$ Department of Textile Engineering, Gaziantep University, Gaziantep, Turkey
}

Corresponding Author: Sümeyye Üstüntağ, sumeyyeustuntag@ erciyes.edu.tr

\begin{abstract}
The aim of this work is to determine the effect of draft ratio and twist level on core visibility ratios of dual-core yarns. For this aim, different types dual-core yarns containing PET/Elastane were produced under controlled conditions. To objectively evaluate the visual quality of the dual-core yarns, the frequency of the core filaments appearing on the yarn surfaces was counted on dyed knitted fabrics produced with these yarns by using image processing algorithm. The results showed that the draft ratio and the twist coefficient were the important factors influencing the core visibility ratios of dualcore yarns. It was found that the core covering performances of the pre-treated dual-core yarns produced with the lowest twist coefficient in the study were more incomplete than the others. Moreover, it was seen that the higher draft ratio on PET filament led to well wrapping of sheath staples to core filaments for dual-core yarns.
\end{abstract}

\section{ARTICLE HISTORY}

Received: 26.10.2019

Accepted: 30.01.2020

\section{KEYWORDS}

Dual-core yarn, pre-treated dual-core yarn, core visibility ratio, draft ratio, twist coefficient

\section{INTRODUCTION}

Core-spun yarn is composite yarn structure developed for utilizing properties of two or more fibre components at the same time with optimum level. The core-spun yarns consist of two different components; a sheath and a core. The core is generally a continuous monofilament or multifilament yarn, which imparts mechanical and functional properties such as strength, dimensional stability, and elasticity to produced core spun yarn. The sheath is staple fibres such as cotton, polyester, viscose etc. and these staple sheaths have been used to improve the wear comfort, in respect of the aesthetic, comfort and handle properties of the resultant yarns [1].

In general, core-spun yarns are classified depending on the raw material used at the yarn centre such as hard-core, softcore. Hard core components such as PET, PA, filaments impart the functional properties such as higher breaking strength, shrinkage and good dimensional stability to resultant core spun yarns. Elastane filaments, as the example for soft core component can exhibit very high elongation (400-800\%) due to their chemical structure. The elastane content even at low usage rates of 3-5\% gives the properties such as shrinkage, better shape fitting, easy maintenance, high elasticity and good recovery to the fabric and garment.

Core-spun yarn properties are mainly influenced by the placement of components in the yarn structure, namely the position of the core filament, the distribution of the sheath staples on the yarn surface, cross sectional configuration, etc. If the core filament is firmly located in the centre of the yarn and well covered by the sheath-staples, the core-spun yarn is evaluated as high quality. Since the eccentric location of the core filament in the yarn axis or irregular covering of the sheath stables lead to bad appearance on the yarn surface, this type of core/sheath distribution is evaluated as yarn defect. Some studies have been

To cite this article: Kılıç G, Yıldırım B, Çelik Hİ, Üstündağ S, Türksoy HG. 2020. The effects of production parameters on the core visibility ratio of dual-core yarns. Tekstil ve Konfeksiyon 30(1), 3-9. 
performed regarding the effect of core-spun yarn structure and spinning parameters on core filament placement and the sheath staples strip-back from core filament phenomena [1-9]. These strip-back phenomena are called as barberpole by Sawhney [2] and it is concluded that strip-back occurs between core and sheath components because of two important reasons; nonconformity between core-spun yarn and core filament linear densities, insufficient twist level. Miao et al. [3] reported that core tension during production has a highly significant effect on sheath slipping resistance. $\mathrm{Su}$ et al. [4] showed that passing the spandex through the $\mathrm{V}$-grooved roller and keeping a contact angle with the front top roller are beneficial to the cover effect of the elastic core-spun yarn. Babaarslan [5] concluded that core positioning has a direct effect on the structure, properties, and performance of core-spun yarns. Kim et al. [6] conceived a measuring system that operates on the machine vision along with programs for recognizing the surface defects and monitoring the measuring process. As a result of the measurement they made with this system, they found that the number of defects has decreases with the increase in twist level. Shanbeh et al. [1] described a method of decreasing the strip-back phenomenon of core-spun yarns. In the proposed method, before the production of these yarns, the core part was coated with a Methyl Methacrylate (MMA) binder using a purpose-built method based on the conventional pad-dry-cure (PDC) system. The obtained results showed the effectiveness of the method proposed for the improvement of the strip resistance of core-spun yarns after the abrasion process, as well as of the abrasion resistance and pilling of woven fabrics. Örtlek et al. [7] investigated the core visibility ratios of hybrid yarns containing copper wire which were produced with 5 different production methods at three different twist levels by using image thresholding method and they found that the production methods and the twist levels have impact on the core visibility ratio value of the hybrid yarns. Türksoy and Kılıç [8] used image processing technique to determine aesthetic differences of hybrid yarns manufactured by three different production methods (ring core-spun, siro corespun, compact core-spun), and three different yarn counts ( $\mathrm{Ne} \mathrm{18,} \mathrm{Ne} \mathrm{24,} \mathrm{Ne} \mathrm{30)} \mathrm{containing} \mathrm{stainless} \mathrm{steel} \mathrm{(SS)} \mathrm{wire}$ and cotton fibres. Based on the core visibility ratio values of the core spun yarns, the best aesthetic performance in terms of hiding the stainless steel was obtained by siro core-spun method and Ne 18 count core-spun yarns.

In recent years, a new type of multicomponent core-spun yarn called as dual-core yarn has been developed in order to overcome a number of problems resulting from the high recovery property of elastane. Dual-core yarns are comprised of two core filaments; an elastane filament and a multifilament (such as PET, PA, PBT or T400®), covered by a staple sheath $[10,11]$. Dual-core yarn production actually has a similar principle to that of the core-spun production. Dual-core yarns can be manufactured on modified conventional ring spinning machine in two different methods [10]. In the first method, previously combined two core threads are fed simultaneously, whereas in the second method, two core threads are fed separately into the centre of sheath fibre bundle. In this study, dualcore yarns produced with the first method were called as pre-treated dual-core (PDC) yarns while the yarns obtained with the second method were named as dual-core (DC) yarns. Many researchers have been focused on core-spun yarn properties [12-18] however very limited number of studies was found on the dual-core yarn properties.

The aim of this study is to determine the effect of draft ratio and twist coefficient on core wrapping quality of dual-core yarns. For this aim, two different dual-core yarns (PDC and DC) containing PET and Elastane were produced with the three different draft ratio and three different twist coefficients. To objectively evaluate the visual quality of the dual-core yarns, the frequency of the core filaments appearing on the yarn surfaces was counted by using image processing technique with a newly proposed approach. Finally, the results were statistically analysed in terms of the significance of independent parameters (draft ratio and twist coefficient) on core wrapping quality.

\section{MATERIAL AND METHOD}

\subsection{Material}

Pre-treated dual-core yarn and dual-core yarn samples having Ne 18/1 count were produced by using Rieter G33 conventional ring spinning machine modified with a positive feed roller system and a V-groove guide. For both yarn types, 100\% Cotton fibers (30 \% Turkmen Sawgin, 50 $\%$ Urfa Rollergin, $20 \%$ USA Sawgin) were used as the sheath staple. The cotton fibers have the following physical properties; $29.21 \mathrm{~mm}$ fiber length, 82.9 UI (Uniformity Index), $32.5 \mathrm{~g} /$ tex strength, $7.91 \%$ elongation and 4.5 micronaire fineness. For the core component of pre-treated dual-core yarns, PET (36 filaments) filament having 55 dtex fineness and Elastane filament (Creora ${ }^{\circledR}$ ) with 44 dtex fineness were welded by intermingling process. Draft was applied to the combined filaments with the ratio of 3.5 during the intermingling process and obtained core component was named as gimped yarn. Gimped yarn was fed to the drafting system and positioned in front roller nip in terms of a standard $\mathrm{V}$-grooved guide roller and covered by cotton fibres (Figure 1a).

Dual-core yarns were produced by using the same core and sheath components having the same fibre properties as pretreated dual-core yarns. However, in dual-core spun yarn production, the core components of PET (36 filaments and 55 dtex) and Elastane (44 dtex) filaments were fed separately under control to the front roller in terms of a standard V-grooved guide roller and covered by cotton fibres (Figure 1b). The production parameters of multicomponent core-spun yarns are given in Table 1 . 


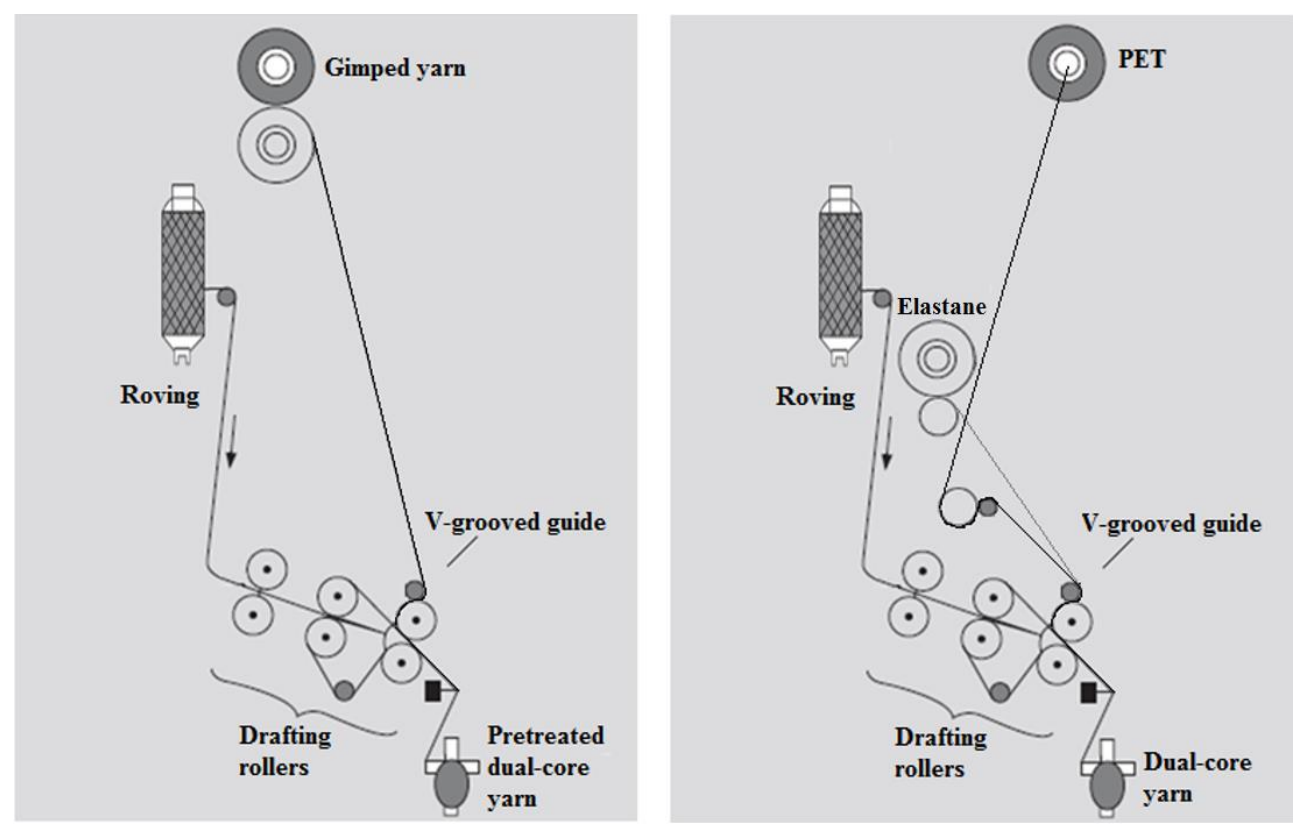

Figure 1. Pre-treated dual-core (a) and dual-core (b) yarn production

Table 1. The production parameters and codes of PDC and DC yarn samples

\begin{tabular}{ccccc}
\hline $\begin{array}{c}\text { Sample } \\
\text { Code }\end{array}$ & $\begin{array}{c}\text { Twist Coefficient } \\
\left(\boldsymbol{\alpha}_{\mathbf{e}}\right)\end{array}$ & $\begin{array}{c}\text { PET draft } \\
\text { ratio }\end{array}$ & $\begin{array}{c}\text { Elastane draft } \\
\text { ratio }\end{array}$ & $\begin{array}{c}\text { Gimped yarn } \\
\text { draft ratio }\end{array}$ \\
\hline PDC1 & 4 & - & - & 1.08 \\
PDC2 & 4 & - & - & 1.03 \\
PDC3 & 4 & - & - & 1.10 \\
PDC4 & 3.5 & - & - & 1.08 \\
PDC5 & 4.5 & - & - & 1.08 \\
\hline DC1 & 4 & 1.08 & 3.5 & - \\
DC2 & 3.5 & 1.08 & 3.5 & - \\
DC3 & 4.5 & 1.08 & 3.5 & - \\
DC4 & 4 & 1.03 & 3.5 & - \\
DC5 & 4 & 1.10 & 3.5 & - \\
DC6 & 4 & 1.08 & 3.3 & - \\
DC7 & 4 & 1.08 & 3.7 & - \\
\hline
\end{tabular}

The visibility of core parts of PDC and DC yarns, which were produced with the different production techniques, was evaluated in the knitted fabric form. As a result of knitted fabric structure, core part of PDC and DC yarns, which is normally invisible, becomes visible. For this reason, single jersey knitted fabrics were manufactured with PDC and DC yarns on sample knitting machine by means of a sample circular knitting machine with 3.5" gauge, 22 feign and one feeder. Since the visibility of the core filament will be determined from the fabric surface, the fabric samples were performed the pre-treatment processes and dyed with reactive dyes. Thus, the cotton i.e. the sheath fibers were colored and the core filament remained as white color without getting dye pigments. So, the measurement success of the developed algorithm was improved. The fabric samples were evaluated with the corresponding yarn sample code in Table 1.

\subsection{Method}

It is considered that the improperly placed filaments or the bad sheath wrapping quality will exhibit different appearance and texture characteristic on the fabric surface. This difference in the fabric texture will result in different pixel values owing to having different light reflection characteristic and different colors. So, the wrapping quality of the dual-core yarns was evaluated in the knitted fabric form by an image processing based method, in this study.

For each yarn sample, seven fabric samples were produced from seven arbitrarily selected yarn copes. Ten image frames were acquired from different parts of each fabric by using Olympus digital microscope with $\times 15$ magnification in ".tiff" format (Figure 2) and totally seventy image frames were obtained for each yarn sample. All lightening conditions and microscope adjustments were kept constant for all image acquisition processes. 


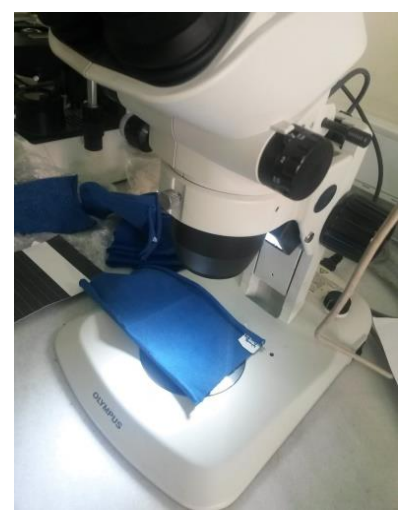

Figure 2. Image acquisition with Olympus digital microscope

The algorithm given in Figure 3 was developed in order to determine the core filament visible ratios from the fabric surface. The algorithm was built in MATLAB environment. Firstly, the image frames acquired in RGB formant was converted to gray scale. RGB images consist of 3-D matrices. Three numerical values are assigned to each pixel in RGB images, each value corresponding to the red, green and blue (RGB) image channel component respectively. Since feature extraction and processing operations are typically applied on the converted grey scale version (2-D) of the image, RGB image frame should be converted to gray scale image using simple transform. Some unwanted external disturbance may occur during the image acquisition operation. These disturbance effects called as noise lead to variation of the signal from its true value in the image processing pipeline [19]. In order to obtain a robust image processing algorithm and achieve the required the success rate, the image frames are dedicated to noise removing filter. In this study, Wiener noise removing filter [20] was applied to reduce the disturbance effects from the image. Then, the image frame was converted to binary form by applying appropriate threshold level. The threshold value is determined by trial and error method. The binary image has two logical values; 1 (white) and 0 (black). The logical conversion of each pixel value is achieved regarding to being higher or lower than threshold value. In this study, the bad covered core filaments were seen on the fabric surface and they were converted white color after logical binary transformation. The other segments well wrapped by fibers were seen as black color. In order to differentiate foreground pixels (white) from the background pixels (black), erosion morphological operation is applied. In the erosion operation, the centre pixel of the structuring used as square in this study element was placed on each foreground pixel value 1. If any of the neighborhood pixels is background pixels' value 0 , the foreground pixel is switched to the background. Thus, the visible core sections seen on the fabric surface were clearly distinguished. Finally, core visibility ratio $(\%)$ was calculated from the ratio of the foreground pixels' area to the total fabric sample area. The application result of the developed algorithm to a fabric sample image is given in Figure 4.

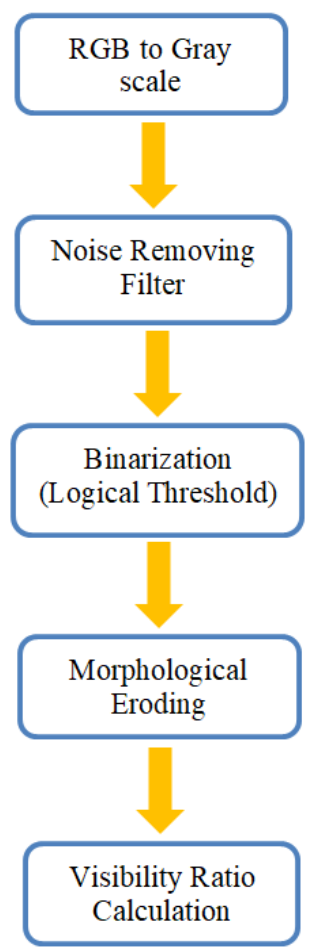

Figure 3. Core visibility measurement algorithm

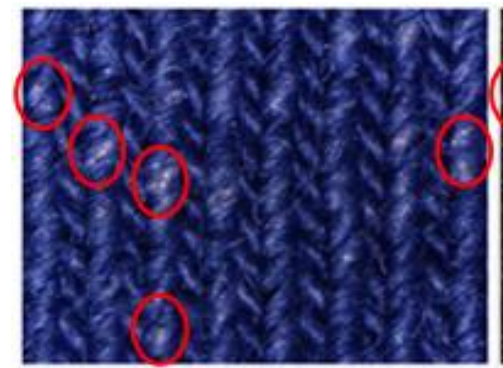

(a) RGB image

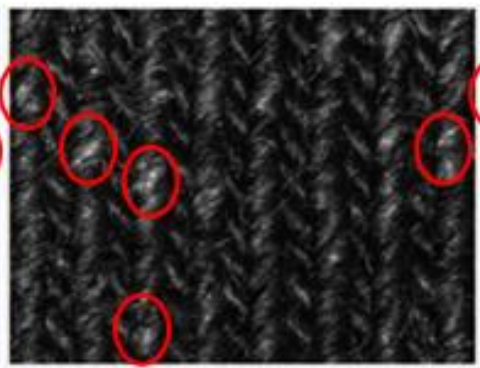

(b) Gray scale image

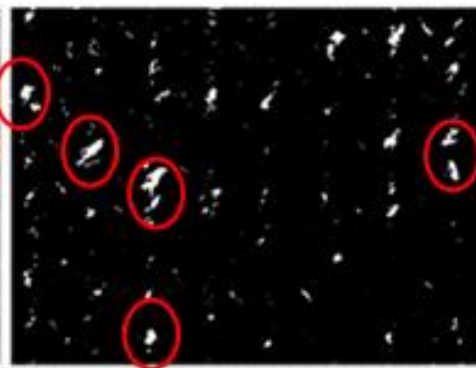

(c) Eroded binary image

Figure 4. The image processing algorithm application 
The significance of independent parameters; draft ratio and twist coefficient were analyzed by using one-way replicated analysis of variance (ANOVA) and the mean differences of subgroups were compared by using DUNCAN post hoc test at 0.05 level using SPSS 13.0 statistical package software.

\section{RESULTS AND DISCUSSION}

The core visibility ratio (CVR) of yarn samples was determined from dyed knitted fabrics by using image processing algorithm. The results were analyzed statistically and submitted in relation to the effect twist coefficient and draft ratio independent parameters. The effect of the twist coefficient and the draft ratio on the core visibility ratio values of the pre-treated dual-core yarns (PDC) and the dual-core yarns (DC) are shown graphically in Figure 5. As seen in Figure 5, the DC6 coded yarn has the lowest core visibility ratio whereas the PDC2 coded yarn has the highest core visibility ratio, in all yarn groups. According to the graph, the DC6 coded yarn produced with 4 twist coefficient, 1.08 PET draft ratio and 3.3 Elastane draft ratio can be said to well cover by the sheath-staples and to be the ideal yarn for core covering performance. In addition, when the graph is evaluated in general, it is seen that higher quality yarns in terms of high core coverage can be obtained with dual-core yarns according to the pretreated dual-core yarn structure. It thought that higher bulkiness resulted from feeding with intermingling process of PET and Elastane filament may affect the core covering performance of pre-treated dual-core yarns negatively.

\subsection{Analysis of Core Visibility Ratio of Pre-treated Dual-Core Yarns}

In order to determine the significance of twist coefficient and draft ratio on core visibility or core covering properties of pre-treated dual core-spun yarn samples, variance analysis (ANOVA) of the measurement results were performed. According to ANOVA test, twist coefficients (tc); $\alpha_{\mathrm{e}}=3.5, \alpha_{\mathrm{e}}=4.0$ and $\alpha_{\mathrm{e}}=4.5$ and gimped yarn draft ratios (dr); 1.03, 1.08 and 1.1 have statistically significant effects $\left(\mathrm{p}_{\mathrm{tc}}=00.040\right.$ and $\left.\mathrm{p}_{\mathrm{dr}}=0.002\right)$ on core visibility ratio of pretreated dual-core yarn samples in $95 \%$ confidence interval. In order to compare the core visibility ratio results of the pre-treated dual-core yarn samples and determine which twist coefficient and gimped yarn draft ratio provide statistically significant difference, multiple comparison test (DUNCAN) was done (Table 2).

The DUNCAN test results of PDC1, PDC4 and PDC5 yarn samples are given in Table 2 for twist coefficient. As observed from the Table 2, there is no significant difference between the core visibility ratio of PDC1 and PDC5 yarn samples. However, there is a significant difference between PDC4 and other two samples; PDC5, PDC1 yarn samples. The highest core visibility ratio is seen in the PDC4 coded yarn that is produced with the lowest twist coefficient $\left(\alpha_{e}=3.5\right)$. The twist coefficient/level is an important factor affecting the frequency with which the core filaments show on the yarn surface. The position of the core filaments can be more frequently alter with the level of the twist coefficient. At a very low twist coefficient, twists seem to allow the core spun yarn to have a migration effect mainly due to the position change magnitude [6]. Moreover, Gharahaghaji et al. [21] stated that using a high level of twist helps to build up the required cohesion between the sheath and the core component. It is thought that the higher the CVR value of the pre-treated dual-core yarns produced with the lowest twist coefficient is related to these situations.

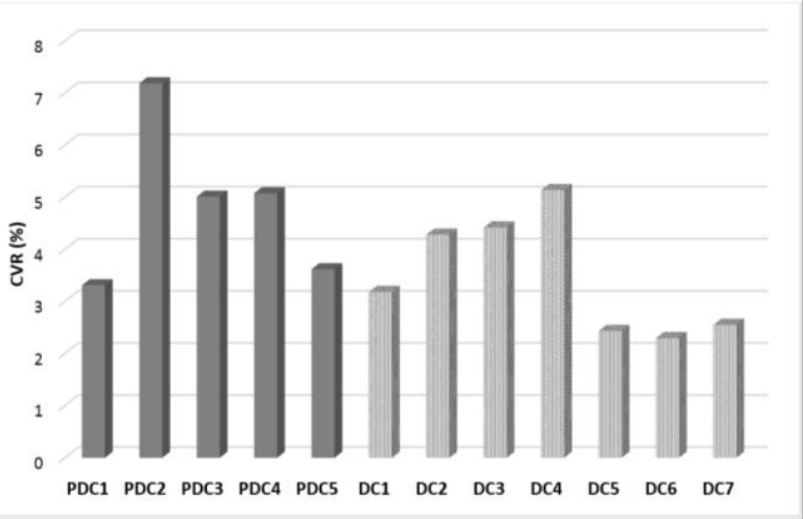

Figure 5. The average core visibility ratio values for pre-treated dual-core yarns (PDC) and dual-core yarns (DC)

Table 2. DUNCAN test results for core visibility ratio of pretreated dual-core yarns with regard to twist coefficient and draft ratio

\begin{tabular}{lccc}
\hline Process & N & \multicolumn{2}{c}{ Subset } \\
\hline Twist Coefficient $\left(\boldsymbol{\alpha}_{\mathrm{e}}\right)$ & & 1 & 2 \\
\hline PDC1 $\left(\alpha_{\mathrm{e}}=4\right)$ & 7 & 3.31 & \\
PDC5 $\left(\alpha_{\mathrm{e}}=4.5\right)$ & 7 & 3.62 & \\
PDC4 $\left(\alpha_{\mathrm{e}}=3.5\right)$ & 7 & & 4.93 \\
Significance Level & & 0.625 & 1.000 \\
\hline Gimped Yarn Draft Ratio & & & \\
\hline PDC1 (1.08) & 7 & 1.93 & \\
PDC3 (1.1) & 7 & 2.57 & \\
PDC2 (1.03) & 7 & & 5.20 \\
Significance Level & & 0.458 & 1.000 \\
\hline
\end{tabular}

When Table 2 is examined in terms of gimped yarn draft ratio, it is seen that there is a significant difference between PDC2 and other two samples; PDC1, PDC3. There is no significant difference between PDC1 and PDC3 samples. The lowest core covering performance is seen in the PDC2 coded yarn sample having 1.03 gimped yarn draft ratio. On the other hand, the best core wrapping performance is obtained with 1.08 core draft ratio among three different draft ratios. 


\subsection{Analysis of Core Visibility Ratio of Dual-Core Yarns}

According to ANOVA result, the twist coefficient (tc), Elastane and PET draft ratios (edr and pdr) have significant effect $\left(\mathrm{p}_{\mathrm{tc}}=0.011, \mathrm{p}_{\mathrm{edr}}=0.0001\right.$ and $\left.\mathrm{p}_{\mathrm{pdr}}=0.001\right)$ on core visibility ratio of dual-core yarn samples. The DUNCAN test results of dual-core yarns according to the twist coefficient, Elastane and PET draft ratios are given in Table 3.

Table 3. DUNCAN test results for core visibility ratio of dualcore yarns with regard to twist coefficient, Elastane and PET draft ratios

\begin{tabular}{lccc}
\hline Process & N & \multicolumn{2}{c}{ Subset } \\
\hline Twist Coefficient $\left(\boldsymbol{\alpha}_{\mathrm{e}}\right)$ & \multicolumn{3}{c}{1} \\
\hline DC1 $\left(\alpha_{\mathrm{e}}=4\right)$ & 7 & 3.18 & \\
DC2 $\left(\alpha_{\mathrm{e}}=3.5\right)$ & 7 & & 4.29 \\
DC3 $\left(\alpha_{\mathrm{e}}=4.5\right)$ & 7 & & 4.42 \\
Significance Level & & 1.000 & 0.737 \\
\hline Elastane Draft Ratio & & & \\
\hline DC6 (3.3) & 7 & 2.30 & \\
DC7 (3.7) & 7 & 2.55 & \\
DC1 (3.5) & 7 & & 3.18 \\
Significance Level & & 0.229 & 1.000 \\
\hline PET Draft Ratio & & & \\
\hline DC5 (1.1) & 7 & 2.44 & \\
DC1 (1.08) & 7 & 3.18 & \\
DC4 (1.03) & 7 & & 5.14 \\
Significance Level & & 0.236 & 1.000 \\
\hline
\end{tabular}

As submitted in DUNCAN test for twist coefficient (Table 3 ), there is no significant difference between DC2 and DC3 yarn samples. However, there is a significant difference between DC1 and other two samples; DC2 and DC3. Also, it can be said that that the highest core covering performances for the pre-treated dual-core and dual-core yarns are obtained with the middle twist coefficient $\left(\alpha_{e}=4\right)$. As shown Table 2 and 3, for both yarn types, the highest twist coefficient $\left(\alpha_{\mathrm{e}}=4.5\right)$ resulted in staple fiber slippage over the filament fibers. It is thought that as core filaments are fed on the side of the spinning zone due to high twist, the wrapping of the sheath staples around the core filaments for both dual-core yarn type is not well and uniform.

When Table 3 is examined for elastane draft ratio, it is seen that there is no significant difference between DC6 and DC7 samples. On the other hand, DC1 results are significantly different from DC7 and DC6 samples. According to Table 3, it can be said that the highest core covering performance is obtained with DC6 coded dualcore yarn that is produced with 3.3 elastane draft ratio.

As shown in the Table 3 , it is seen that there is no significant difference between DC5 and DC1 dual-core yarn samples in terms of PET draft ratio. It means that the draft ratio changes from 1.08 to 1.1 does not have significant effect on core wrapping level of sheath staple.
However, DC4 sample is significantly different from DC1 and DC5 dual-core yarn samples. There is decreasing tendency in core visibility results of dual-core yarn samples with the increase in PET draft ratio value. This may be due to the fact that as the PET draft ratio is increased, the linear density of PET core component is getting finer, number of cotton sheath fibers increase and the core wrapping performance of the sheath fibers increase.

\section{CONCLUSION}

The aim of this study is to evaluate the aesthetic defects of different dual-core yarns containing PET/Elastane by using image processing technique in terms of production parameters. In order to determine the core visibility ratios of the pre-treated dual-core yarns (PDC) and dual-core yarns (DC) produced with different twist coefficient and draft ratio, single jersey knitted fabrics were manufactured from these yarns. Thanks to the knitted fabric structure and colored sheath fibers, core part of PDC and DC yarns became visible. Then, image processing technique was applied to the dyed knitted fabric samples acquired by using Olympus digital microscope and thus the image of the core filaments on the yarn surface became clear.

The results showed that the pre-treated dual-core yarns produced with the lowest twist coefficient $\left(\alpha_{\mathrm{e}}=3.5\right)$ had the highest core visibility ratio. It can be revealed that the lower amount of twist on yarn structure leads to inadequate wrapping of sheath staples to core filaments. Moreover, this result indicates that the interlacing between the core filaments and the sheath staples decreases due to low twist coefficient and staple fiber slippage over the filament takes place more intensively. For pre-treated dual-core yarn samples, it was seen that the highest and lowest core covering performances were obtained with 1.08 and 1.03 core draft ratios, respectively. On the other hand, there was not any increasing or decreasing tendency in core visibility ratio with core draft ratio increase or decrease. So, it can be stated that the optimum core draft ratio is 1.08 for pretreated dual-core yarn samples.

Since the dual-core yarn samples were produced by feeding two core filaments; Elastane and PET, the draft effect of these two core filaments were evaluated separately. For dual-core yarns, it was revealed that the core covering performance on the yarn surface improved with increase in PET draft ratio and the highest core covering performance was obtained with 1.1 PET draft ratio. This mean that the lower PET draft ratio leads to incomplete core coverage and may result in end breaks in the next stages of processing. When the effect of elastane draft ratio was investigated, it was seen that the highest core wrapping performance was obtained with 3.3 draft ratio. Apart from these, the results showed that the twist factor at $4\left(\alpha_{\mathrm{e}}\right)$ gave the optimum core covering performances. 


\section{REFERENCES}

1. Shanbeh M, Baghaei B, Alidadi S, Tabibi A. 2011. Coating of core yarn. An alternative method of decreasing the strip-back phenomenon of core-spun yarns. Fibres \& Textiles in Eastern Europe 19, 5(88), 28-32.

2. Sawhney APS, Kimmel LB. 1992. Improved method of producing a cotton covered/polyester staple-core yarn on a ring spinning frame. Textile Research Journal 61(19), 21-25.

3. Miao M, How YL, Ho SY. 1996. Influence of spinning parameters on core yarn sheath slippage and other properties. Textile Research Journal 66(11), 676-684.

4. Su C, Maa M, Yang H. 2004. Structure and performance of elastic core-spun yarn. Textile Research Journal 74(7), 607-610.

5. Babaarslan O. 2001. Method of producing a polyester/viscose core spun yarn containing spandex using a modified ring spinning frame. Textile Research Journal 71(4), 367-371.

6. Kim HJ, Kim JS, Lim JH, Huh Y. 2009. Detection of wrapping defects by a machine vision and its application to evaluate the wrapping quality of the ring core spun yarn. Textile Research Journal 79(17), 1616-1624

7. Örtlek HG, Çalışkan Ç, Kurban R. 2013. A comparative study on the physical properties of hybrid yarns containing copper wire. Journal of Textiles and Engineer 20(89), 11-20.

8. Turksoy HG, Kılic G. 2016. Evaluation of core visibility ratio for hybrid yarns using image processing technique. Tekstil ve Konfeksiyon 26(2), 147-152.

9. Miao M, Barnes S, Vuckovic L. 2010. High-speed video graphic study of filament-core yarn spinning. The Journal of the Textile Institute 101(3), 242-252.

10. Bedez Ute T. 2018. Analysis of mechanical and dimensiona properties of the denim fabrics produced with double-core and corespun weft yarns with different weft densities with double-core and core-spun weft yarns with different weft densities. The Journal of the Textile Institute 110(2), 179-185.

11. Zhang H, Xue Y, Wang S. 2006. Effect of filament over-feed ratio on surface structure of rotor-spun composite yarns. Textile Research Journal 76(12), 922-923.
12. Hau T, Wong NS, Tang WM. 2018. Study on properties of elastic core-spun yarns containing a mix of spandex and PET/PTT bicomponent filament as core. Textile Research Journal 88(9), 10651076

13. Celik P, Bedez Üte T, Özden D, Çömlekçi H, Akkale EC. 2009. Öz/Manto oranı ve büküm sayısının filament özlü ipliklerin iplik özelliklerine etkisi. Tekstil Teknolojileri Elektronik Dergisi 3(2), 2937 .

14. Herath CN, Kang BC. 2008. Dimensional stability of core spun cotton/spandex single jersey fabrics under relaxation. Textile Research Journal 78(3), 209-216.

15. Pourahmad A, Johari MS. 2009. Production of core-spun yarn by the three-strand modified method. The Journal of the Textile Institute 100 (3), 275-281.

16. Yang HW, Kim HJ, Zhu CH, Huh Y. 2009. Comparisons of core sheath structuring effects on the tensile properties of high-tenacity ring core-spun yarns. Textile Research Journal 79(5), 453-460

17. Qadir MB, Hussain T, Malik M, Fahemm A, Jeong SH. 2014. Effect of spandex linear density and draft ratio on the physical and mechanical properties of core-spun cotton yarns. The Textile Institute 105(7), 753-759.

18. Erez E, Çelik P. 2014. A research on the parameters of the affecting yarn properties of cotton-polyester rigid core-spun yarns. Textile and Apparel 24(2), 195-201.

19. Solomon C, Breckon T. 2011. Fundamentals of digital image processing a practical approach with examples in MATLAB Chichester, UK: John Wiley \& Sons Ltd, 200-202.

20. Sonka M, Hlavac V, Boyle R. 2008. Image processing, analysis and machine vision international student edition, 3rd. ed. New York: Springer, 661-665.

21. Gharahaghaji A., Zargar EN, Ghane M, Hossaini A. 2010. Clusterspun yarn - A new concept in composite yarn spinning. Textile Research Journal 80(1), 19-24. 